\title{
A Novel Algorithm Between Fuzzy Number's Distance in Facility Location
}

\author{
Elahe Abdoos ${ }^{1, *}$, Alimohamad Ahmadvand ${ }^{2}$, Hossein Eghbali ${ }^{1}$ \\ ${ }^{1}$ Department of Industrial Engineering, University of Eyvanekey, Eyvanekey, Iran \\ ${ }^{2}$ University of Eyvanekey, Eyvanekey, Iran
}

Email address:

elaheabdous@gmail.com (E.Abdoos),m.ahmadvand@eyc.ac.ir(A. Ahmadvand), h.eghbali@eyc.ac.ir(H. Eghbali)

${ }^{*}$ Corresponding author

\section{To cite this article:}

Elahe Abdoos, Alimohamad Ahmadvand, Hossein Eghbali. A Novel Algorithm Between Fuzzy Number's Distance in Facility Location. International Journal of Management and Fuzzy Systems. Vol. 4, No. 3, 2018. pp. 53-56. doi: 10.11648/j.ijmfs.20180403.13

Received: June 19, 2017: Accepted: September 14, 2018: Published: September 19, 2018

\begin{abstract}
Measuring the distance is one of the most important components in planning industrial units. Since human words and reasons are vague and imprecise, the distance between fuzzy numbers in most industrial units is required in real-world decision-making and planning. In many cases, ranking occur in fuzzy conditions which obtained information is uncertain, thus it creates a possibility of confusion for the designer in ranking problems. In this study, first the importance and application of distance in industrial units ranking and expressed some ranking methods are dealt, then a new algorithm will be provided for the distance between two fuzzy numbers which is more precise and quicker than previous methods. The proposed method can be a very suitable management strategy to implement it in reality.
\end{abstract}

Keywords: Locating, Industrial Units Planning, Fuzzy Set Theory, Fuzzy Numbers Distance

\section{Introduction}

One of the most important issues in the field of industrial engineering is the industrial units planning. Although it is clear that in today's industrial environment, consumption experience and relying on trial and error cannot guarantee success in contrast to past, because now scientific principles have associated with industrial work which industry is not just art anymore. Nowadays, artisans cannot be succeed by just relying on their taste and experiences learned from their mentors in the workplace and stay pursuant in their industry (Samadi, Karimi-sani, 2010).

Since human words and reasons are vague and imprecise, distance between fuzzy numbers in most stages of decision - making and planning of industrial units in the real world is required. Professor Lotfi Asgarzadeh first time in 1965 (Asgharzadeh, 1965) provided such a tool by introducing the theory of fuzzy sets and fuzzy logic. Distance of fuzzy numbers are one of the most important tools in the engineering industry, in particular management decisionmaking. Fuzzy values have many applications in real problems. So far, most proposed methods cannot reasonably calculate all modes of fuzzy numbers. The fact is that the distance of fuzzy numbers cannot be measured like real numbers. Although fuzzy numbers are not easily measured because their distance are vague and imprecise but so far many methods in this regard are suggested and discussed by the researchers. Each of the proposed methods for a certain category of numbers (normal, abnormal, Positive fuzzy numbers and negative fuzzy numbers) can be used. It is important that proper technique is used in the right conditions.

In this study, the importance and application of distance in the industrial units ranking and outlines some of the methods are first studied, then a new algorithm for distance between two fuzzy numbers will be provided in which is more precise and quicker than previous methods. The proposed method can be a very suitable management strategy to implement it in reality. However, fuzzy numbers distance cannot be easily measured because they are vague and imprecise distance, but so far many methods in this regard suggested and discussed by the researchers. 


\section{General Theory of Fuzzy Sets}

In relation to the predicate logic, set theory is raised and each set with its members are fully identified, or in other words time set is fully introduced due to consider each element certainly is a member or is outside of the set. Each set has a clear characteristic of its own. Criteria of elements membership in a set is the identifier attribute of that set and if each member has that attribute, and if there are no attribute, it is known as off-set. This membership criteria is called as membership function (or characteristic function).

Definition (fuzzy sets): Assume that $X$ is a reference set. The indicator function of each non-fuzzy subset $A$ of $X$ to $\{0,1\}$ is defined as follows.

$$
\mu_{A}(x)=\left\{\begin{array}{lll}
1 ? & \text { if } & x \in A \\
0 ? & \text { if } & x \notin A
\end{array}\right.
$$

Definition (membership function): If we extend the range of indicator function from the two members set of $\{0,1\}$ to the range of $[0,1]$, we will have a function that relates a number from interval $[0,1]$ to each $x$ of $X$. This function is called membership function $\tilde{A}$. This is not a common set anymore, but is called fuzzy set. Therefore, a fuzzy set $\tilde{A}$ is a subset that the degrees of membership of their members can be acquired continuously from $[0,1]$. This set is completely and uniquely determined by a membership function which is shown with $\mu_{\tilde{A}}(x)$. In this case, $\mu_{\tilde{A}}(x)$ is a function that grants a number of the interval $[0,1]$ to each one the element of $X$ as the membership degree of that element in the fuzzy set a. $\mu_{\tilde{A}}(x)$ value closeness to a number indicates more belonging of $X$ to the fuzzy set $X$ and vice versa its closeness to zero indicates less belonging of $X$ to the fuzzy set $\tilde{A}$.

Definition (fuzzy set): If $X$ is the reference set which show each of its members with $x$, fuzzy set $\tilde{A}$ of is expressed by ordered pairs as follows:

$$
\tilde{A}=\left\{\left(x, \mu_{\tilde{A}}(x)\right) \mid x \in X\right\} \infty
$$

Membership function or the membership degree of fuzzy set shows the belonging to this set. The range of this function is a nonnegative real numbers which we consider one as its maximum value and in normal situation is 0 and 1 respectively.

Definition (fuzzy number): fuzzy number $\tilde{\mathrm{A}}=(\mathrm{a}, \mathrm{b}, \mathrm{c}, \mathrm{d} ; \mathrm{w})$ is defined as a sub-phase space of real numbers $\mathrm{R}$ with membership function of $\mu_{\tilde{\mathrm{A}}}(\mathrm{x})$ which is contained of following features (ghazanfari and Rezaei, 1385 Abbasbandy and Asady, 2006).

(a) $\mu_{\tilde{\mathrm{A}}}$ is a continuous function from $\mathrm{R}$ with interval of $[0, w]$ which $0 \leq w \leq 1$.

(b) $\mu_{\tilde{A}}(x)=0$ And $\forall x \in(-\infty, a]$. (c) $f_{\tilde{A}}$ In the interval of $[a, b]$ is absolutely ascending.

(d) $\mu_{\tilde{A}}(x)=w$ And $\forall x \in[a, b]$ where $w$ is a constant number and $0 \leq w \leq 1$.

(e) $\mu_{\tilde{A}}$ In the interval of $[c, d]$ is absolutely descending.

(f) $\mu_{\tilde{A}}(x)=0$ And $\forall x \in[d,+\infty)$

Note: If $b$ and $c$ were not coincide, fuzzy number is trapezoidal otherwise it is triangular. And if and $a=b$ then fuzzy number $A$ is a distance or range. Also, if $a=b=c=d$ then fuzzy number $A$ is a real number.

Definition (Parameter display of fuzzy number): Fuzzy number $u(r)$ which is indicate by an ordered pair of functions as $(\underline{u}(r), \bar{u}(r))$ and $0 \leq r \leq 1$, is called parameter display $u(r)$ that applies in the following conditions.

(1) $u(r)$ is a continuous bounded function from left and non-increasing on the interval $[0,1]$.

(2) $u(r)$ is a continuous bounded function from left and non-decreasing on the interval $[0,1]$.

(3) $\underline{u}(r) \leq \bar{u}(r)$ and $\forall r ; 0 \leq r \leq 1$.

Fuzzy number $\tilde{A}$ is called positive (negative) and will be shown by $(\tilde{A}<0 \tilde{A}>0)$, if its membership function would be $\mu_{\tilde{A}}(x)$, then for each $x<0$ (each ) meet the requirement in $\mu_{\tilde{A}}(x)=0$ condition.

Definition (fuzzy number): a fuzzy number is called a fuzzy number if:

$$
\mu_{\bar{A}}(x)= \begin{cases}L\left(\frac{a-x}{\alpha}\right), & x \leq \alpha, x>0 \\ R\left(\frac{x-a}{\beta_{\tilde{A}}}\right), & x \geq a, \beta>0 .\end{cases}
$$

Where $a$ is the peak of $\tilde{A}$ and $a$ and $\beta$ are the left width and right width respectively and function $L(0)$ which is the left side function, applies on following relations.

(1) $L(x)=L(-x)=0$.

(2) $L(0)=1, L(1)=0$

(3) $L(x)$ at $[0, \infty]$ is non-increasing.

And the right side function $R(0)$ define as well as $L(0)$.

By using the peak and left and right width functions and sides functions, fuzzy number $\tilde{A}$ is shown as below.

$$
\tilde{A}=(a, \alpha, \beta)_{L R}
$$

Clearly $\tilde{A}=(a, \alpha, \beta)_{L R}$ is positive and $\tilde{B}=(b, \gamma, \delta)_{L R}$ are equal if and only if $a=b$ and $\alpha=\gamma$ and $\beta=\delta$.

On the other hand, since each fuzzy number is a set, we can define it a subset as follows.

A fuzzy number $L R$ is called as fuzzy number subset $\tilde{B}=(b, \gamma, \delta)_{L R} \quad$ like $\tilde{A}=(a, \alpha, \beta)_{L R} \quad$ if and only if $a-\alpha \geq b-\gamma$ and $a+\beta \leq b+\delta$. 


\section{Introduce a New Algorithm for Distance Between Fuzzy Numbers}

In this section, a new algorithm for distance between two fuzzy number is introduced and then next season will be focused on its implementation and prove it and based on the proposed method, an algorithm for industrial units ranking will be offered.

Assume that $\tilde{\mathrm{A}}=\left(x_{1}, x_{2}, x_{3}\right)$ and $\tilde{B}=\left(y_{1}, y_{2}, y_{3}\right)$ are two triangular fuzzy numbers, also consider the distance between $\tilde{\mathrm{A}}$ to $\tilde{B}$ as $\tilde{D}_{\tilde{A} \tilde{B}}$ which will be $\tilde{D}_{\tilde{A} \tilde{B}}=\left(d_{1}, d_{2}, d_{3}\right)$.

In the following an algorithm will be offered to calculate the distance between two arbitrary triangular fuzzy numbers as $\tilde{\mathrm{A}}$ and $\tilde{B}$ that are the same $d_{1}, d_{2}$ and:

First step:

(1) Compare $x_{1}$ and $y_{1}$.

(2) If $x_{1}=y_{1}$ then $d_{1}=\left|x_{1}-y_{3}\right|$ and proceed to next step, otherwise the next level will be checked.

(3) Compare $x_{2}$ and $y_{2}$.

(4) If $x_{2} \geq y_{2}$ then $d_{1}=\left|x_{1}-y_{3}\right|$ and proceed to next step, otherwise the next level will be checked.

(5) If $x_{2} \prec y_{2}$ then $d_{1}=\left|y_{1}-x_{3}\right|$ and proceed to next step.

Second step:

1. Calculate $d^{\prime}=\left|x_{2}-y_{2}\right|$.

2. Equal $d^{\prime}$ and $d_{1}$ maximum to $d_{2}$ in this way $d_{2}=\max \left\{d^{\prime}, d_{1}\right\}$ then proceed to third step.

Third step:

1. Compare $x_{3}$ and $y_{3}$.

2. If $x_{3}=y_{3}$ then $d_{3}=0$ and it is over, otherwise $d_{3}=\max \left\{y_{3}-x_{1}, x_{3}-y_{1}\right\}$.

Prove the Proposed Algorithm

Proposition 1: The proposed method has metric property:
(1) $\tilde{D}_{\tilde{A} \tilde{B}} \geq 0$.
(2) $\tilde{D}_{\tilde{A} \tilde{B}}=\tilde{D}_{\tilde{B} \tilde{A}} \cdot$
(3) $\tilde{D}_{\tilde{A} \tilde{C}} \leq \tilde{D}_{\tilde{A} \tilde{B}}+\tilde{D}_{\tilde{B} \tilde{C}}$.

\section{Implementation of Algorithm}

\section{Benefits of Proposed Distance Algorithm}

This section outlines some of the benefits of the proposed distance between two fuzzy numbers. For this purpose, using the numerical examples, the results of the proposed method and other methods will be compared.

\section{Benefit 1}

The distance between zero and fuzzy number a by Chakraborty and Chakraborty approach (Chakraborty and Chakraborty, 2006) and the Guha and Chakraborty (Guha and Chakraborty, 2010) has flaw, because in these two methods, the answer will not be a which means $b$, that this answer does not seem reasonable, however, by using proposed method, distance between a fuzzy number and zero will be equal to the number which is $\mathrm{s}$, that is a more reasonable answer and this is a very important benefit of the proposed method.

Benefit 2

The following table is obtained by data given by using Sadi-Nezhad et al paper (Sadi-Nezhad, 2012). The results of distance $\mathrm{d}$ is shown using proposed method and other methods in table 2. According to Table 2, in some cases, results of Voxman (Voxman, 1998), Guha and Chakraborty (Guha and Chakraborty, 2010) and Sadi-Nezhad et al (SadiNezhad et al., 2012) methods are not reasonable. This example demonstrates the benefits of the proposed method against other methods.

Table 1. $A_{i}$ Triangular fuzzy numbers.

\begin{tabular}{ll}
\hline $\boldsymbol{i}$ & $\boldsymbol{A}_{\boldsymbol{i}}$ \\
\hline 1 & $(0,2,3)$ \\
2 & $(-1,0,1)$ \\
3 & $(-3,-2,-1)$ \\
4 & $(-5,-4,0)$ \\
\hline
\end{tabular}

Table 2. Results Comparison.

\begin{tabular}{llllll}
\hline Case & & $\boldsymbol{D}_{\text {Sadi-nezhad }}$ & $\boldsymbol{D}_{\text {Voxman }}$ & $\boldsymbol{D}_{\text {Guhd et al. }}$ & $\boldsymbol{D}_{\text {proposed }}$ \\
\hline 1 & $D_{A_{1} A_{1}}$ & $(0,0,3)$ & $(0,0,3)$ & $(0,0,3 / 2)$ & $(0,0,0)$ \\
2 & $D_{A_{1} A_{2}}$ & $(0,2,4)$ & $(2,2,4)$ & $(1 / 2,2,3)$ & $(1,2,4)$ \\
3 & $D_{A_{1} A_{3}}$ & $(1,4,6)$ & $(1,4,6)$ & $(5 / 2,4,5)$ & $(1,4,6)$ \\
4 & $D_{A_{1} A_{4}}$ & $(0,6,8)$ & $(0,6,8)$ & $(3,6,7)$ & $(0,6,8)$ \\
5 & $D_{A_{2} A_{2}}$ & $(0,0,2)$ & $(0,0,2)$ & $(0,0,3 / 2)$ & $(0,0,0)$ \\
6 & $D_{A_{2} A_{3}}$ & $(0,2,4)$ & $(0,2,4)$ & $(1,2,3)$ & $(0,2,4)$ \\
7 & $D_{A_{2} A_{4}}$ & $(0,4,6)$ & $(4,4,6)$ & $(5 / 2,4,5)$ & $(1,4,6)$ \\
8 & $D_{A_{3} A_{3}}$ & $(0,0,2)$ & $(0,0,2)$ & $(0,0,1)$ & $(0,0,0)$ \\
9 & $D_{A_{3} A_{4}}$ & $(0,2,4)$ & $(2,2,4)$ & $(0,2,3)$ & $(3,3,4)$ \\
\hline 10 & $D_{A_{4} A_{4}}$ & $(0,0,5)$ & $(0,0,5)$ & $(0,0,5 / 2)$ & $(0,0,0)$ \\
\hline
\end{tabular}

\section{Conclusion}

The distance between two fuzzy numbers plays an important role in the engineering industry, also widely used in data mining and operations' research. Also measuring distance is one of the most important components in issues of industrial units planning.. Since human words and reasons are vague and imprecise, the distance between fuzzy numbers in most industrial units is required in real-world decisionmaking and planning. In this study, first the importance and application of distance in industrial units ranking and expressed some ranking methods are dealt, then a new algorithm were provided for the distance between two fuzzy numbers which is more precise and quicker than previous methods. The proposed method can be a very suitable management strategy to implement it in reality. 


\section{Footnote}

This article is extracted from a thesis entitled "a new algorithm in industrial units ranking by using the concept of fuzzy numbers distance" written by Elahe Abdoos and doctor Ali Mohammad Ahmadvand is supervisor and advisor is Hossein Eghbali of Eyvanakey university.

\section{References}

[1] Samadi, MohammadReza, Karimi sani, Mohammad. (2010), factory layout design; Software introduction, Journal of Industrial Engineers comment.

[2] ghazanfari, Mahdi, Rezaei, Mahmoud. (1385). Introduction to the theory of fuzzy sets, Science and Technology University Press.

[3] Abbasbandy, S., and Asady, B. (2006). Ranking of fuzzy numbers by sign distance, Information Sciences, 176, 24052416.

[4] Abbasbandy, S., and Hajighasemi, S. (2010). A Fuzzy Distance between Two Fuzzy Numbers Information Processing and Management of Uncertainty in KnowledgeBased Systems, Applications, E. Hüllermeier, R. Kruse, and F. Hoffmann, Editors, Springer Berlin.

[5] Ali Beigi, M., Hajjari, T., and Ghasem Khani, E. (2015) A New Index For Fuzzy Distance Measure, Inf. Sci. 9, No. 6, 3017-3025.

[6] Bloch, I. (1999). On fuzzy distances and their use in image processing under imprecision, Pattern Recognition, 32, 18731895.

[7] Chakraborty, C., and Chakraborty, D. (2006). A theoretical development on a fuzzy distance measure for fuzzy numbers, Mathematical and Computer Modeling, 43, 254-261.

[8] Chen, S. H., and Hsieh, C. H. (2000). Representation, Ranking, Distance, and Similarity of L-R type fuzzy number and Application, Australia Journal of Intelligent Information Processing Systems, 6 (4), 217-229.

[9] Chen, S. H. and Hsieh, C. H. (1998). Graded mean integration representation of generalized fuzzy numbers. The 6th Conference on Fuzzy Theory and Its Applications (CD-ROM), filename: 031. wdl, Chinese Fuzzy Systems Association, Taiwan, 1-6.
[10] Dubois, D., and Prade, H. (1978). Operation on fuzzy numbers, International Journal of Systems Science, 9, 613-626.

[11] Ghasem Khani, E., and Ali Beigi, M. (2015). A Novel Strategy for Comparative Points in Facility Layout Problem with Fuzzy Logic, International Journal of Management and Fuzzy Systems, 1 (2), 15-20.

[12] Guha, D., and Chakraborty, D. (2010). A new approach to fuzzy distance measure and similarity measure between two generalized fuzzy numbers, Applied Soft Computing, 10, 9099.

[13] Hajjari, T. (2010). Fuzzy Distance Measure for Generalized Fuzzy Numbers, The 11th International Conference on Intelligent Technologies (INTECH), Bangkok, Thailan.

[14] Heilpern, S. (1997). Representation and application of fuzzy numbers, Fuzzy sets and Systems, 91 (2), 259-268.

[15] Kaufmann, A., and Gupta, M. M. (1991). Introduction to fuzzy arithmetic theory and applications, Van Nostrand Reinhold.

[16] Kumar, A., Bahtia, N., and Kuar, M. (2009). A new approach for solving fuzzy maximal flow problems rough sets, fuzzy sets, data mining and granular computing, H. Sakai, et al., Editors, springer Berlin/Heidelberg, 278-286.

[17] Liu, X. (1992). Entropy, distance measure and similarity measure of fuzzy sets and their relations, Fuzzy Sets and Systems, 52 (3), 305-318.

[18] Pedrycz, W. (2007). Collaborative and knowledge-based fuzzy clustering, International journal of Innovating computing, Information and Control, 3 (1), 1-12.

[19] Sadi-Nezhad, S., Noroozi, A., and Makui, A. (2012). Fuzzy distance of triangular fuzzy numbers, Journal of Intelligent and Fuzzy Systems.

[20] Saha, P. K., Wehrli, F. W., and Gomberg, B. R. (2002). Fuzzy Distance Transform: Theory, Algorithms and Applications, Computer Vision and Image Understanding, 86, 171-190.

[21] Tran, L., and Duckstein, L. (2002). Comparison of fuzzy numbers using fuzzy distance measure, Fuzzy Sets and Systems, 130, 231-341.

[22] Voxman, W. (1998). Some remarks on distances between fuzzy numbers, Fuzzy Sets and Systems, 100, 353-365.

[23] Zadeh, L. A. (1965). Fuzzy set, Information and Control, 8, 338-353. 